\title{
Multifunctional Mesostructures: Design and Material Programming for 4D-printing
}

\author{
Tiffany Cheng \\ Institute for Computational Design \\ and Construction \\ Stuttgart, Germany \\ tiffany.cheng@icd.uni-stuttgart.de \\ Benjamin Stolz \\ Freiburg Materials Research Center \\ Freiburg, Germany
}

\author{
Yasaman Tahouni \\ Institute for Computational Design \\ and Construction \\ Stuttgart, Germany
}

Rolf Mülhaupt

Freiburg Materials Research Center

Freiburg, Germany

\author{
Dylan Wood \\ Institute for Computational Design \\ and Construction \\ Stuttgart, Germany
}

\author{
Achim Menges \\ Institute for Computational Design \\ and Construction \\ Stuttgart, Germany
}
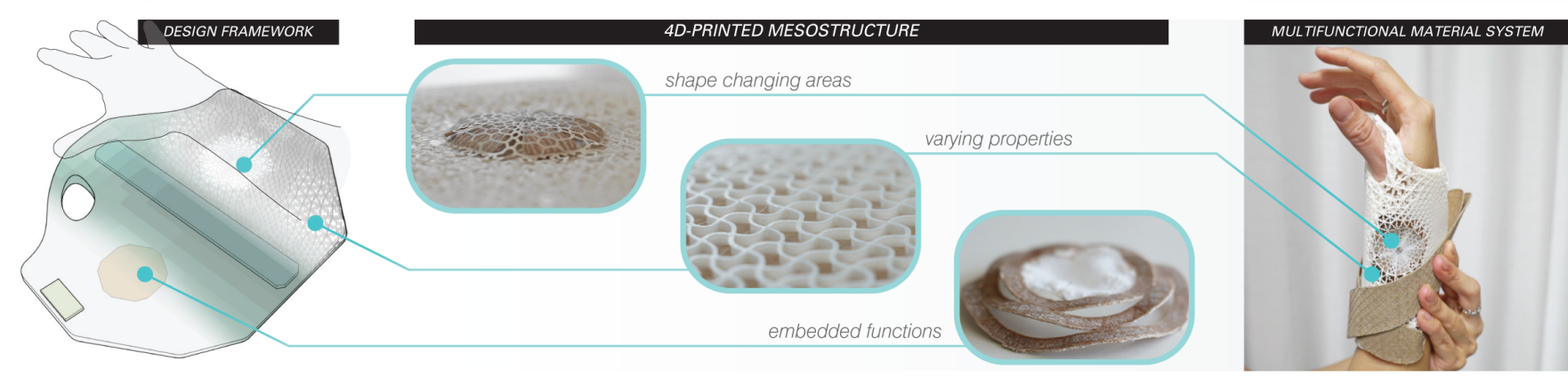

Figure 1: An orthotic device with physically encoded areas of shape change, varying stiffness, and passive cooling.

\begin{abstract}
Natural materials, such as in plant and bone organs, adapt to their surroundings with functionally graded underlying structures. Advances in extrusion-based 4D-printing have enabled the manufacture of bio-inspired systems with varying properties and selfshaping behaviors. However, tailoring the internal composition of such systems relies on specialized knowledge, as most computeraided design $(\mathrm{CAD})$ applications are based on a modeling paradigm that considers objects as surfaces or solids with no geometrical definition of the interior structure. We propose that engineered materials with differentiated and heterogeneous mesostructures can achieve nature-inspired functionality. We present a design approach for tailoring the internal topology of $4 \mathrm{D}$-printed material systems, using intuitive geometric descriptions from existing CAD workflows. We introduce a material programming framework for assigning and tuning material properties such as elasticity and shape change with varying magnitudes and anisotropies throughout a
\end{abstract}

Permission to make digital or hard copies of part or all of this work for personal or classroom use is granted without fee provided that copies are not made or distributed for profit or commercial advantage and that copies bear this notice and the full citation on the first page. Copyrights for third-party components of this work must be honored

For all other uses, contact the owner/author(s).

SCF '20, November 5-6, 2020, Virtual Event, USA

(c) 2020 Copyright held by the owner/author(s).

ACM ISBN 978-1-4503-8170-3/20/11.

https://doi.org/10.1145/3424630.3425418 volume. Our method translates the desired properties into an assembly of functional patterns for fabrication via anisotropic material deposition. To demonstrate this framework, we show several types of material behaviors, including self-shaping double curvature and embedded passive cooling. Finally, we produce a prototype of a wearable assistive device that highlights the integration of multiple functions. Through design and material programming, the resulting 4D-printed material systems underline how nature-inspired mesostructured material networks can be physically encoded with custom-designed behaviors, shape changes, and functionalities.

\section{CCS CONCEPTS}

- General and reference $\rightarrow$ Design; • Applied computing $\rightarrow$ Computer-aided manufacturing.

\section{KEYWORDS}

additive manufacturing, adaptive systems, functional structures, shape change, responsive materials

\section{ACM Reference Format:}

Tiffany Cheng, Yasaman Tahouni, Dylan Wood, Benjamin Stolz, Rolf Mülhaupt, and Achim Menges. 2020. Multifunctional Mesostructures: Design and Material Programming for 4D-printing. In Symposium on Computational Fabrication (SCF '20), November 5-6, 2020, Virtual Event, USA. ACM, New York, NY, USA, 10 pages. https://doi.org/10.1145/3424630.3425418 


\section{INTRODUCTION}

In nature, materials address a variety of performance requirements with intricate, underlying structures. The femur bone and conifer cone are two examples of hierarchically organized structures with exceptional mechanical properties. The femur, which must support almost the full weight of the human body, is burdened not only with compression but also tension, as loads are applied transversely from the hip socket rather than directly along the axis of the bone [Rudman et al. 2006; Wolff 1870]. The internal bone structure plays an important role in the overall functional properties, and indeed, studies have shown that changes in both arrangement and orientation occur in response to specific loading conditions, such as during pregnancy or extended stays in space [Lang et al. 2006]. The scale of a Pinaceae conifer cone is another famous instance of an adaptive, structured tissue which autonomously responds to environmental stimuli with reversible movements. The differentiated morphology of hygroscopic tissues with different swelling and shrinking properties [Dawson et al. 1997; Harlow et al. 1964] allows pine cones to passively open and close in response to relative humidity $(\mathrm{RH})$ even as a dead plant organ, millions of years after detaching from the tree [Poppinga et al. 2017].

Recent advances in polymer chemistry and computational fabrication have enabled the engineering of mechanisms with graded compliance [Howell 2013; Megaro et al. 2017; Saggere and Kota 1999] and adaptation without any electro-mechanical control or operating energy [Menges and Reichert 2015; Reyssat and Mahadevan 2009]. Additive manufacturing (AM) has made it possible to modulate various properties and deformation behaviors [Frenzel et al. 2017; La Magna and Knippers 2018]; it is even possible to embed further functionality through specialized circuitry, such as externally sourced resisters and transistors [Lewis et al. 2016; Valentine et al. 2017]. Moreover, the emergence of smart materials that can be processed by extrusion-based AM has allowed the production of adaptive structures that can interact with their surroundings without the need for discrete sensors, actuators, or controllers [Boley et al. 2019; Gladman et al. 2016; Yao et al. 2015]. Termed 4D-printing [Tibbits 2014], the technique for tailoring passive shape changes using fused filament fabrication (FFF) machines has led to new material systems with heat and moisture responsiveness [An et al. 2018; Correa et al. 2015; Wang et al. 2017].

Complexity is inexpensive for biological systems; while the adage "complexity is free" [Lipson and Kurman 2013] is often said about AM, capitalizing on the affordances of this technology to produce complex shapes as well as tailor their internal compositions relies on specialized knowledge and training. Most 3D computer-aided design (CAD) programs are based on the surface modeling paradigm known as boundary representation (BREP), which considers only the surfaces or outer shell of an object. As BREPs represent the limits of a solid, they are thus unable to convey spatial variations within the interior. There have been efforts to introduce the concept of modeling variable material distributions represented by volumetric voxels [Michalatos and Payne 2016], though this method relies on advanced 3D-printers that can handle the mixing of multiple materials. Harnessing the promises of AM necessitates the ability to represent material distributions throughout a $3 \mathrm{D}$ volume, while allowing their fabrications on machines which are accessible.

\subsection{Key contributions}

We propose a design and material programming framework for dealing with the complexity of integrating multiple functions (such as self-shaping and variation of material properties) in a simplified way. The core contribution of this paper is a unified workflow to tailor 4D-printed material systems with differentiated and heterogeneous mesostructures - accessible using readily available, off-the-shelf printers and filaments, but also highly customizable by allowing specialty materials and externally sourced components.

We formulate a method for tuning the internal topology of these material systems, using the intuitive and well-established geometric modeling approach of existing non-uniform rational basis spline (NURBS) workflows. By designing different functional regions and specifying their physical metadata, we can program material properties and behaviors, such as elasticity and shape change. Based on these user-prescribed parameters, the resultant kinematics of this networked assembly of functional regions can be visualized. This model representation also serves as the basis for structuring the material system's mesostructure [Raney and Lewis 2015], achieved using FFF. Through additive fabrication and choreographed paths of material deposition, the objects become physically encoded with their programmed properties. Finally, the framework enables functionally specialized systems to be designed by allowing externally sourced components to be directly embedded in the 4D-printed material system. Our main contributions are the following:

- A material programming toolkit of active, passive, and external functional regions which can be geometrically defined and customized with physical metadata.

- A model representation for generating topologies well suited for visualization and extrusion-based AM.

- The translation of behaviors and properties into a functionally patterned material network with mesostructured features.

- The fabrication logic and sequence for producing internal mesostructures via FFF, and embedding externally sourced components during the extrusion process.

This design framework is demonstrated through a series of case studies that showcase both single and double curvature self-shaping, as well as the embedding of materials sourced from external processes. In addition, we produce prototypes of assistive technologies for medical applications, highlighting the integration of multiple functions. Finally, we discuss the performance benefits of highly differentiated and heterogeneous assistive wearable devices with specific behaviors, shape changes, and functionalities.

\subsection{Related work}

Fabricating objects with variable properties offer a wide range of benefits. The carpal skin glove [Oxman 2010], a 3D-printed prototype for a wrist splint to protect against carpal tunnel syndrome, demonstrated the mapping of a particular patient's pain-profile to the distribution of hard and soft materials. While this design has illustrated the performance benefits of personalized medical devices, the use of highly advanced and expensive equipment for commercial AM has played a role in restricting the transfer of this approach in both research and application. 
Existing literature on meta-material mechanisms has shown that it is possible to tune mechanical properties using consumer 3Dprinting equipment. Research conducted on tileable cell structures has demonstrated that varying degrees of flexibility can be achieved [Amorim et al. 2019; Ion et al. 2016; Panetta et al. 2015]. Despite the intricate patterning, however, the use of generic slicing software for generating the 3D-printing toolpaths still limits the resolution of achievable properties, as the majority of slicers consider objects to have isotropic interior infill structures within their outer shells.

The extrusion-based process of FFF inherently produces anisotropy through the paths of material deposition. The field of $4 \mathrm{D}$-printing has exploited the inherent anisotropy produced by this method to construct self-shaping material systems structured at the mesoscale [Correa et al. 2020; Vazquez et al. 2020; Wood et al. 2016]. Though these works have employed anisotropic extrusion to create bioinspired shape change, the literature indicates difficulty in organizing the complex arrangement of multiple materials at the mesoscale, especially in achieving design heterogeneity beyond the functional abstraction of bilayers. As a result, 4D-printed mechanisms have remained largely monofunctional.

Other researchers have investigated functional gradation from the perspective of materials science, showing that modulation of chemical composition can achieve a high degree of functional, mechanical, and optical differentiation [Giachini et al. 2020; MogasSoldevila et al. 2014]. While custom-engineered materials offer a wide range of novel functionalities, the fabrication of liquid and paste materials necessitates highly specialized equipment, with specific processing requirements depending on the material itself.

\section{DESIGN \& MATERIAL PROGRAMMING}

Our framework offers a hierarchical workflow that greatly simplifies the design process, visualized in Figure 2. This hierarchy abstracts the complexity required to translate a conceptual selfshaping design to the specific machine code for FFF.

Thus, one can design the self-shaping behaviors using intuitive tools: the substructure of the object is designed by defining functional regions and specifying their physical metadata. This high-level design is then used to generate a model representation, which provides an indication of the shape change and allows for a rapid feedback loop to quickly iterate designs. The model is then translated into functional patterns, which become the $4 \mathrm{D}$-printed mesostructure networks through the $4 \mathrm{D}$-printing sequence \& logic.

\subsection{Functional regions \& physical metadata}

To tailor and modulate the internal composition of an object, we simplify the material system into a networked assembly of smaller sub-units called functional regions.

Using familiar geometric descriptors from CAD modeling applications, these functional regions can be defined with closed NURBS curves on the XY-plane. Our parametric model, developed within a visual programming environment (Grasshopper3D running on Rhinoceros3D), allows the specification of physical metadata associated with each functional region.

We formulate three types of regions with distinct functions: the active region, passive region, and external region. Each of their physical metadata contains parameters which can be tuned in terms of the anisotropy and amount.

The active regions are characterized by a bilayer structure, and self-shape by producing single curvature bending. The compounding of the two layers, composed of a stimuli-responsive actuating material and a relatively stable restricting material, translates differential volume change upon stimuli response into a bending behavior. While any combination of two filaments with a differential between their stimuli-responsiveness can be employed, we demonstrate this

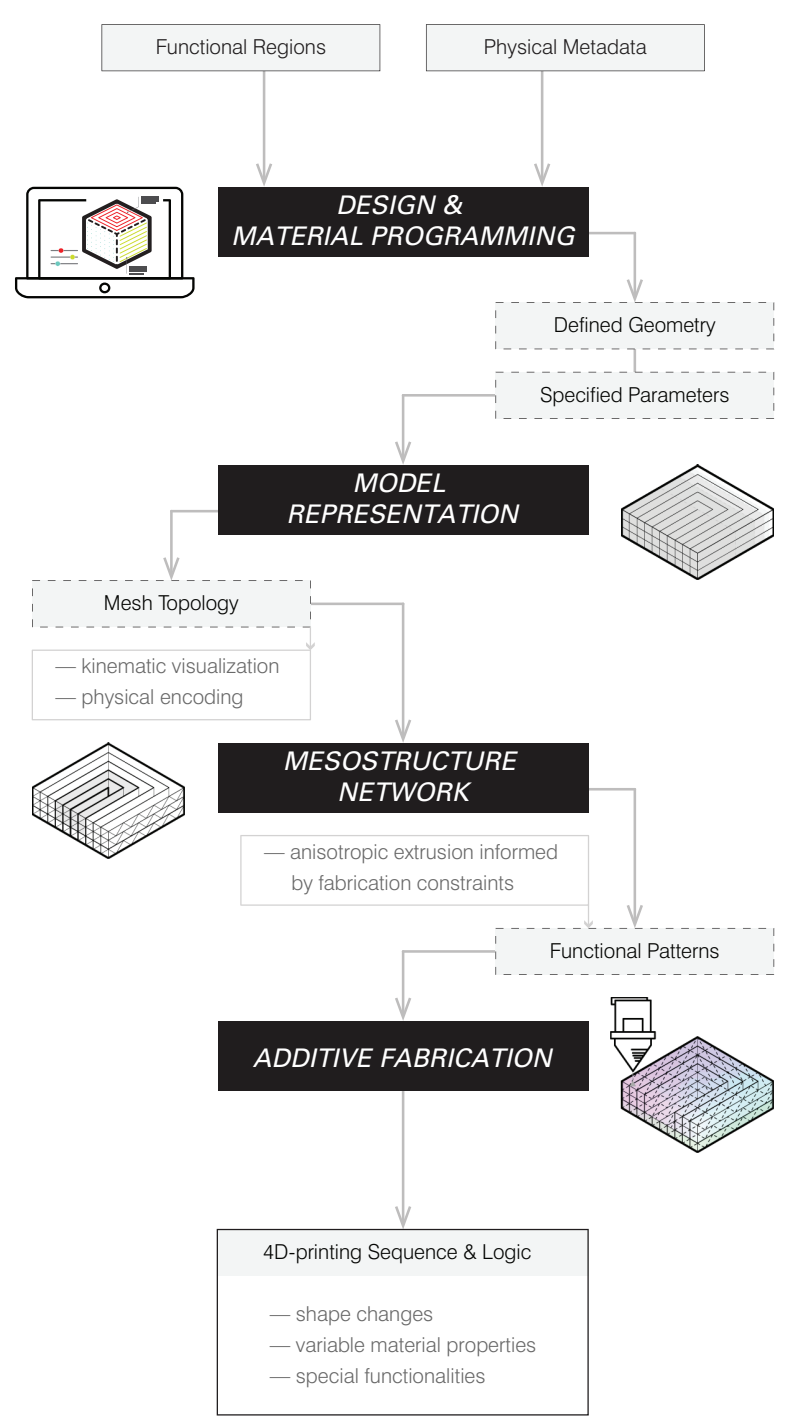

Figure 2: Multifunctional material systems are abstracted as a networked assembly of functional regions, each of which can be defined with physical metadata. These simple inputs are then translated into a differentiated mesostructure, which can be $4 \mathrm{D}$-printed with the parameters and sequence required to produce the designed properties and behaviors. 


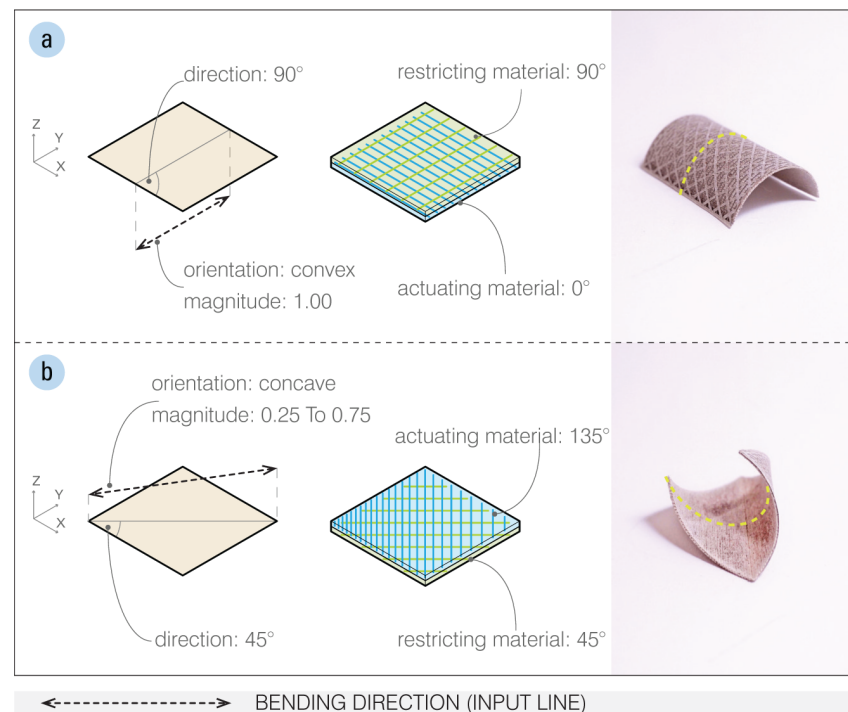

Figure 3: The geometric definition and material programming of an active region with (a) convex, constant bending and (b) concave, variable bending at an angle. The restricting material is extruded along the bending direction (input line), while the actuating material is extruded perpendicular to it.

approach using hygroscopic filaments which respond to moisture. A single line provides an intuitive interface for adjusting the bending orientation, magnitude, and direction. As shown in Figure 3, the line is projected onto the active region and the distance between sample points along the input line and projected line determines the bending orientation (sign of Z-value), magnitude (absolute Z-value), and direction (angle of the projected line on the XY-plane).

The passive regions are physically programmed with mechanical properties such as stiffness and Poisson effect. Using the control points of a NURBS surface as the user interface (Figure 4a), these properties can be varied and distributed throughout an object; the distances between both sets of sample points on the control surface and planar surface of the passive region determine the property's value. This allows the easy manipulation of heightmaps (Figure 4b) in 3D-space, which are correlated to the material property gradients (Figure 4c) of the designed object and its function (Figure 4d). While unable to change shape on their own, the passive region can be encoded to allow stretching and contraction of the material in-plane (whereas the active region will bend a planar surface out-of-plane in only one direction). When combined, the functional variation of active and passive regions enables double curvature to be produced in $4 \mathrm{D}$-printed self-shaping material systems.

The external regions enable high customizability by allowing specialized, externally sourced parts to be embedded inside the $4 \mathrm{D}$ printed material system. Non-filament materials and components manufactured from external processes can be represented by an abstract, closed surface. Additionally, the amount of embedding (that is, how much of it is exposed or enclosed) can be assigned with a percentage value.

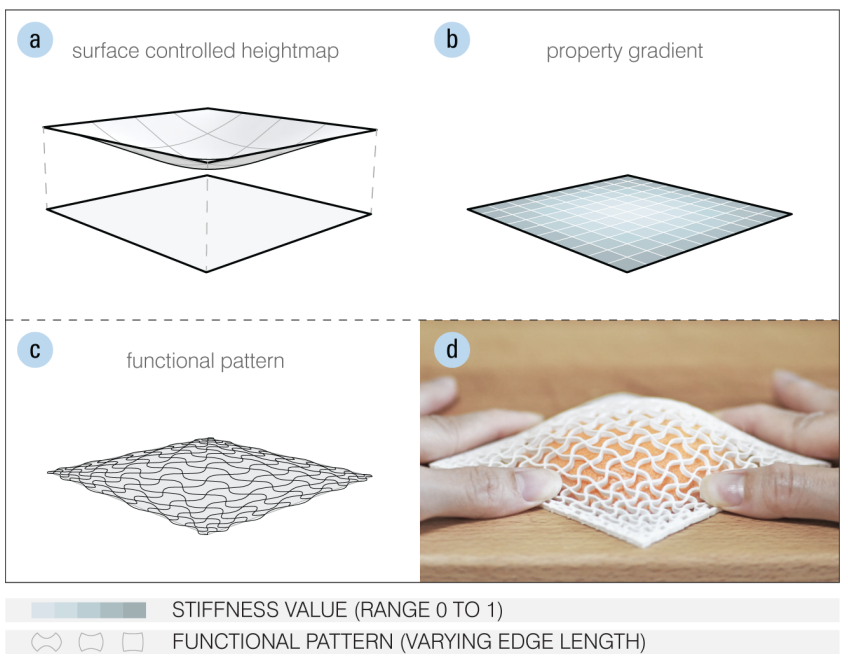

Figure 4: (a) Using the control surface, varying elasticities can be designed across an object. (b) This property gradient is represented in the model as a heightmap containing multiple values, (c) which are mapped to the functional patterns which physically encode the desired elasticity. (d) Demonstrated here on a cut grapefruit is the resultant differentiated stretching.

\subsection{Model representation}

Based on user-prescribed parameters, the model is generated with a topology suited for transfer to both kinematic visualization and extrusion-based fabrication. The topology is broadly discretized according to its functional regions and physical metadata as a structured grid. In a networked assembly of different functional regions, the physical metadata determines the grid directionality in each region. The grid is aligned according to the bending directions of the active regions, or stretching and contraction directions of the passive regions. From this grid, the mesh is then generated using a brute-force planar search for bounding regions [Alfredo Ferreira et al. 2003].

The predicted shape change can then be visualized using a solver based on shape matching constraints [Müller et al. 2005] implemented with the Shape0p library and the Kangaroo2 live physics engine. Again, the physical metadata determines the target shape for optimization. The bending orientation, direction, and magnitude determine the directional scaling constraints, which are applied to the mesh vertices of the active regions. Mesh vertices in the passive regions will preserve their distances and spatial relationships to varying degrees depending on the programmed properties (stiffness and Poisson effect). This implementation provides a qualitative indication of how user-specifications for multiple functional regions at the local level impacts the way an object deforms globally, offering an intuitive interface for users to interactively explore the design space. 


\subsection{Mesostructure network}

This mesh topology serves as a blueprint for creating a material network with mesostructured features. It is the multi-material organization of the mesostructure that dictates the system's overall behavior and properties.

The process of FFF preserves the fibrous architecture of the filament, and thus allows us to tailor the mesostructure by controlling various aspects of the material deposition - including the trajectory, speed, and rate of extrusion. The geometry of the mesostructure is formed by the trajectory of extrusion, which leaves anisotropic material paths. Depending on the functional regions and their physical metadata, these trajectories follow different patterns (Figure 5).

The physical metadata that can be defined for active regions, comprised of two materials layers, is the bending orientation, bending direction, and bending magnitude. The bending orientation is encoded by spatial ordering: convex bending is achieved by extruding the actuating layer first and then the restricting layer over it (Figure 3a), while concave bending is achieved by the opposite ordering (Figure $3 \mathrm{~b}$ ). The bending direction is controlled by the anisotropy of both layers, achieved by the angle of extrusion paths on the $X Y$-plane; the extrusion trajectory of the restricting material follows the bending direction, while the actuating material is extruded perpendicular to that direction.

The bending magnitude is influenced by the thickness (that is, the number of layers in the Z-axis) and porosity (the gaps between material depositions in the XY-plane) within both material layers. More thickness and less porosity in each layer will generally increase the flexural rigidity and result in less bending. By adjusting the porosity in the restricting layer, flexural rigidity can be tuned even without adjusting the thickness. In the actuating layer, the thickness and porosity also impact the actuation time. Thus, the bending magnitude and actuation time can additionally be tuned by modulating the resultant ratio between actuating and restricting layers; as the restricting layer has a negligible effect on actuation time, this allows the actuation time and bending magnitude to be controlled independently.

The passive regions are produced with functional patterns that physically encode the desired material properties. These patterns can be instantiated on a rectilinear or triangular grid, corresponding to the mesh model.

Stiffness is modulated by adjusting the pattern geometry along the mesh edges, which act as mechanisms for encouraging or preventing both stretching and contraction. Wavy structures arise from increasing the length of a pattern's edge (without altering the overall size of the pattern); when the pattern is pulled, its effective volume can expand until the wavy structures are fully straightened. The lengths to be added along the edges are extracted from the values obtained from the property gradient map, illustrated in Figure 4 . The Poisson effect can also be tailored using well-established principles and patterns from the field of meta-material mechanisms [Kolken and Zadpoor 2017; McCaw and Cuan-Urquizo 2018], and the effect of changing other parameters such as the geometry can be explored.

The external regions are left as voids during the initial extrusion process. Once the externally-sourced part is embedded, the extrusion process can be continued to secure it in place. In addition,

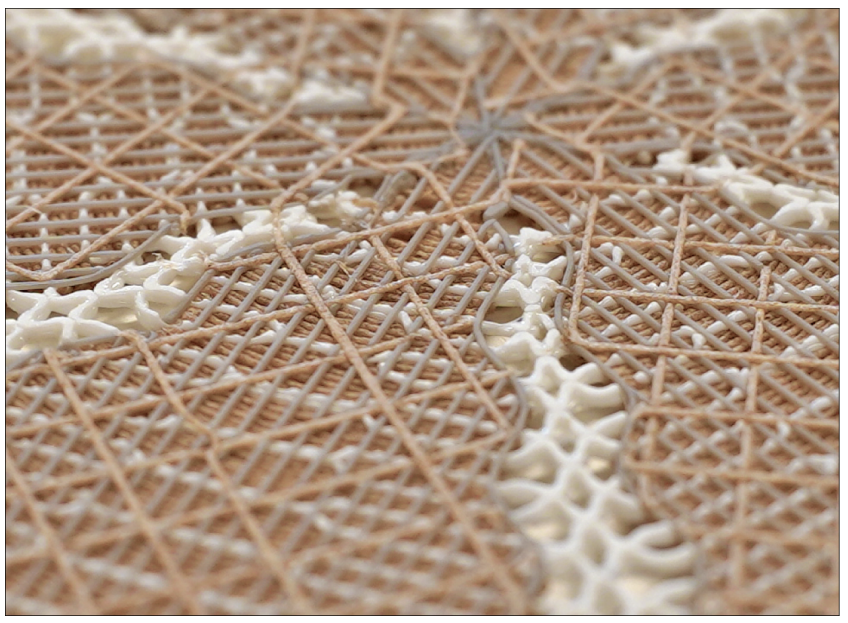

Figure 5: The material system's different regions are interconnected by a fibrous, mesoscale network of multiple interwoven materials. The passive regions (white) stretch and contract in conjunction with the self-morphing active regions (brown).

the rate of extrusion can be increased to fully enclose the part, or decreased to expose a certain portion of it.

\subsection{Additive fabrication}

The mesostructure's trajectories, along with the details regarding the speeds and rates of extrusion, are then translated into numerical control machine language (Voxel2GCode). This fabrication data can be executed by any consumer desktop FFF 3D-printer capable of reading G-code commands, and instructs the machine on where to move, at what speeds, and with what material as well as how much. The qualities of the material paths are informed by the combination of speed and rate of extrusion. For this study, we kept a constant speed and adjusted the rate of extrusion to increase (or decrease) the amount of material deposition. Our extrusion parameters using 0.35 $\mathrm{mm}$ diameter brass nozzles produced material paths with adjustable widths ranging from $0.45 \mathrm{~mm}$ to $1.5 \mathrm{~mm}$. The equipment we used (FELIX Tec 4 Dual Head 3D-printer, FELIX, Utrecht, Netherlands) allowed us to extrude mesostructure details at $0.05 \mathrm{~mm}$ precision.

The active regions require just that two filament materials with different expansion coefficients be used. In this study, we used a combination of hygroscopic wood-polymer composite (LAYWOODmeta5, Lay Filaments, Cologne, Germany) extruded at $190^{\circ} \mathrm{C}$, and acrylonitrile butadiene styrene (ABS, MakerBot Industries, New York, USA) extruded at $200^{\circ} \mathrm{C}$. The hygroscopic filament will equalize with the surrounding $\mathrm{RH}$; therefore, the material storage and fabrication environment should be kept relatively stable so that, while printing the initially flat geometries, shape change is not induced. The 4D-printed material systems can transform from flat to form (and vice versa) within the span of hours when switching from $\mathrm{RH}$ extremes (25\%-90\%), which was tested in a climate-controlled box (MiniOne Humidity Generator, Preservatech, Bydgoszcz, Poland). When submerged in water, the transformation can be observed in the span of minutes. For the passive regions, we employed a 


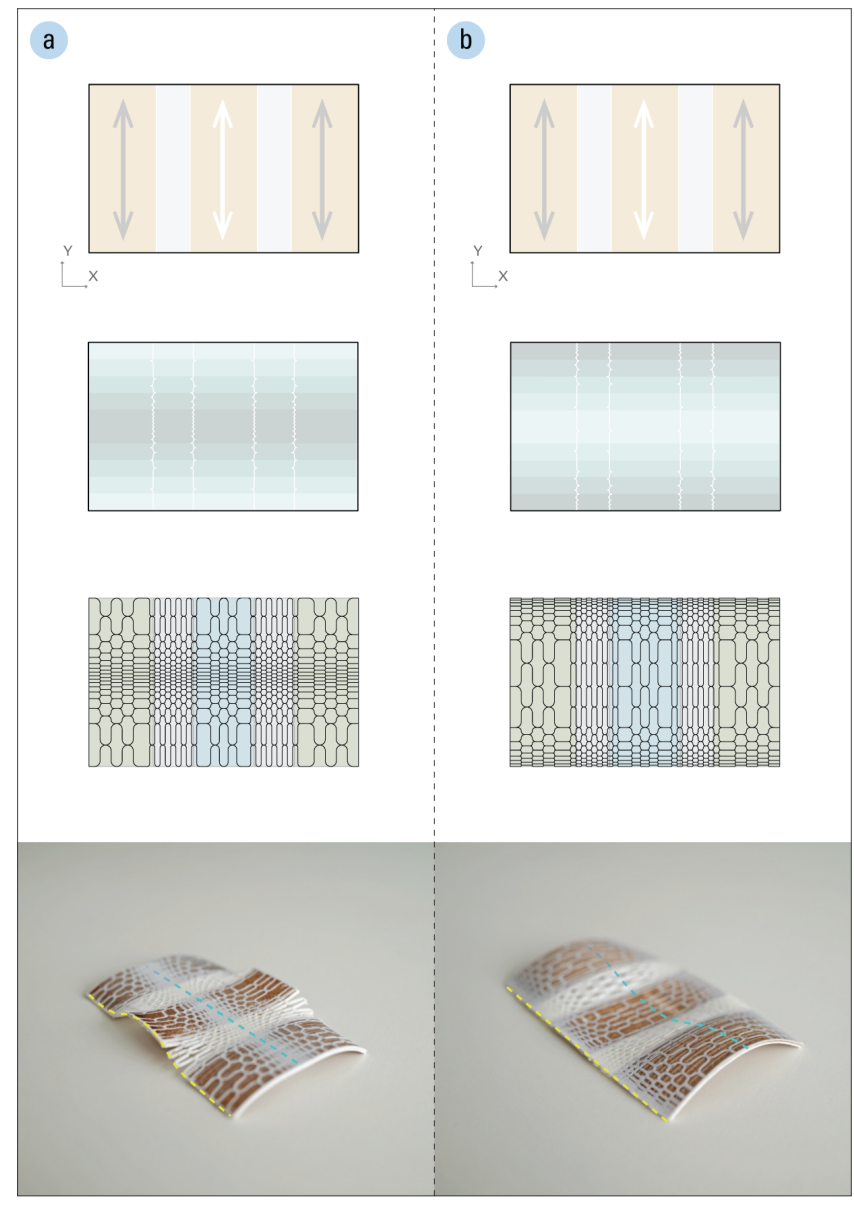

Figure 6: A parameter study on the impact of passive regions on global self-shaping. Both samples contain identical active regions but different property gradients for the passive regions and, as a result, differentiated $4 \mathrm{D}$-printed mesostructures. Sample (a) is programmed with low elasticity along the spine and high elasticity at the edges, resulting in functional patterns that allow stretching at the edges. The opposite is programmed for sample (b).

thermoplastic copolyester (FlexiFil, FormFutura, Nijmegen, Netherlands), with a shore hardness of 45D and tensile modulus of 95 $\mathrm{MPa}$, extruded at $210^{\circ} \mathrm{C}$. All filaments were $1.75 \mathrm{~mm}$ in diameter and printed onto a bed heated to $45^{\circ} \mathrm{C}$.

\section{CASE STUDIES}

We now illustrate the advantages of our design framework through three case studies. First, we systematically explore the design space of networked active and passive regions for self-shaping with double curvature, a property that is particularly challenging to integrate in 4D-printed systems with hygroscopic materials. Second, we demonstrate the use of external regions to embed specialty devices for passive cooling within a $4 \mathrm{D}$-printed self-shaping mechanism. Finally, we show a full workflow using our framework to design a customized orthotic splint with integrated shape changing areas.

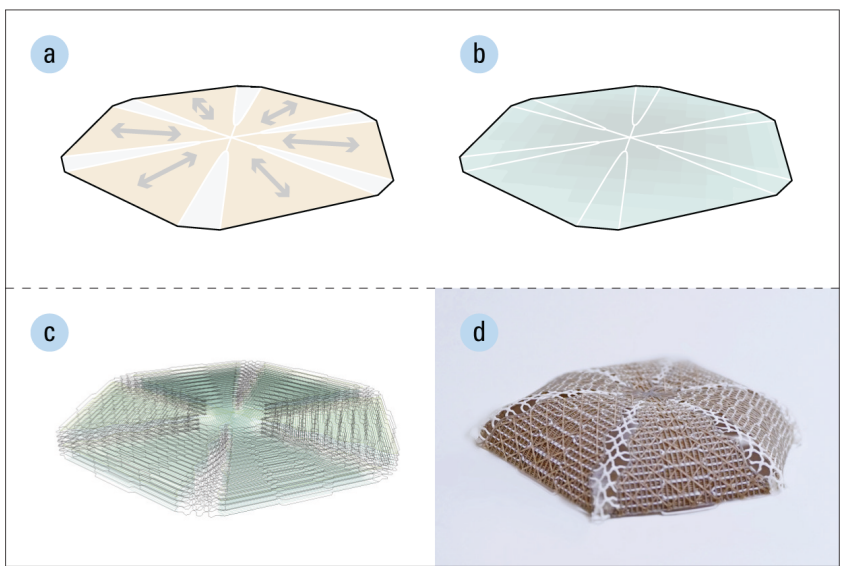

Figure 7: (a) The dome is designed with radially arrayed active regions. (b) The passive regions are programmed with low elasticity in the center and high elasticity at the edges. (c) During the self-shaping process, the mesostructure allows for more contraction at the edges. (d) The resultant 4Dprinted material system shows a synclastic geometry.

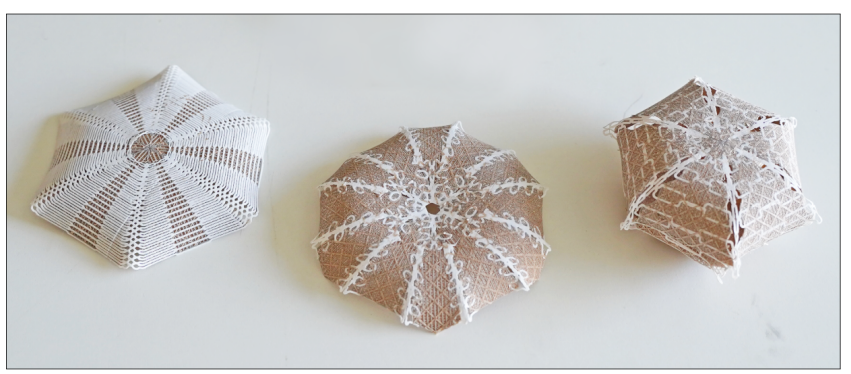

Figure 8: Variations of synclastic geometries were formed using (left) passive regions with low elasticity, (middle) more and smaller segments of active regions, and (right) high elasticity in the passive regions.

\subsection{Double curvature self-shaping}

While single curvature self-shaping can be achieved with active regions alone, double curvature cannot: bending a flat surface in two directions (and back) requires regions of the surface to simultaneously grow and contract in different directions and amounts. Double curvature self-shaping 4D-printed systems can be achieved by strategically combining active and passive regions, and the geometry can be further tailored by programming the property gradients of the passive regions. However, how the functional pattern gradients of the passive regions affect the resulting shape in combination with the active regions is non-trivial. While creating specific shapes typically requires a lot of trial and error, our framework allows us to quickly and systematically explore the design space.

As an example, we consider the effects of elasticity gradients, and thus differentiated stretching, on double curvature. The base design is a sheet formed by three active regions with alternating bending orientations, shown in Figure 6, joined by passive regions. We then test various gradients by modulating the density of the functional 


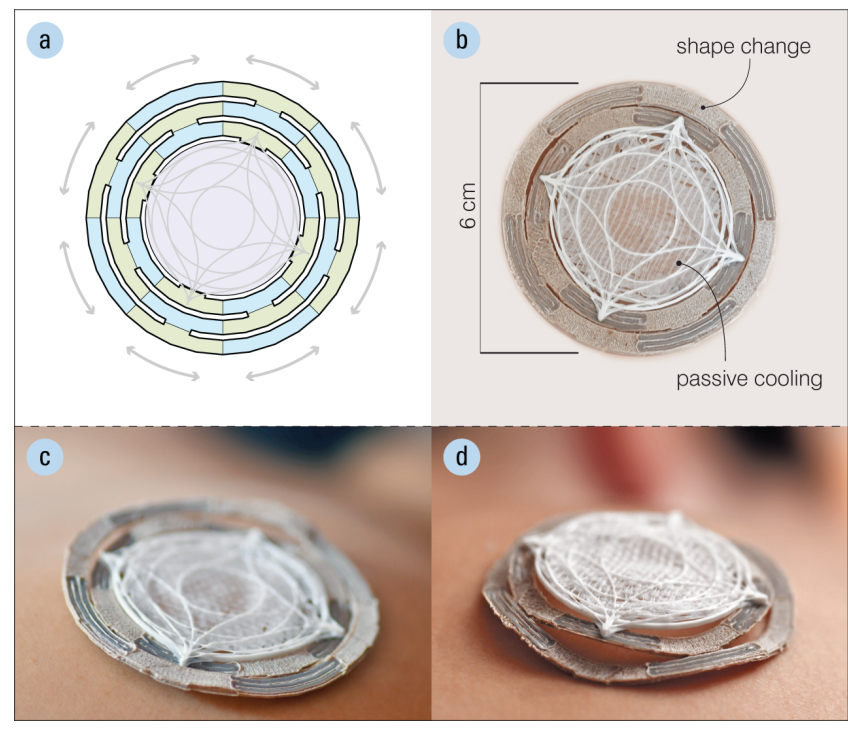

Figure 9: (a) The design for a 4D-printed on-body device. (b) The passive cooling function is integrated with shape change. (c) The patch at high RH with full contact on the skin. (d) The patch at low RH, after 4 hours, with the passive cooling element lifted.

pattern across the $\mathrm{Y}$-axis, to get a qualitative assessment of how this property affects the final shape. We find that, by forming areas with high or low pattern density, thereby influencing the local elasticity, we can control where the surface is allowed by the mesostructure to stretch and expand. The pattern allows more deformation where there is less density at the edges (Figure 6a); whereas, the pattern will allow more deformation along the spine when there is less density there (Figure 6b).

We also investigate the strategic integration of active and passive regions in creating a synclastic geometry. This assembly is constructed from six triangular active regions with radially arranged bending directions (Figure 7a), connected by six passive regions. The passive regions are functionally patterned with high density in the center and low density at the edges (Figure 7b), producing a differentiated mesostructure which allows contraction at increasing magnitudes from the center to the edge (Figure 7c). The 4D-printed combination of active and passive regions successfully formed into a synclastic dome shape (Figure 7d). By tuning the number of active regions and their anisotropic bending properties, combined with varying gradients of in-plane elasticity in the passive regions, a wide variety of self-shaping domes can be $4 \mathrm{D}$-printed, as shown in Figure 8.

\subsection{Embedding specialty functions}

While we can $4 \mathrm{D}$-print complex, adaptive self-shaping structures by programming active and passive regions, we use external regions to embed devices with specialty functionality that cannot be printed. We show that it is possible to incorporate the functionality of passive cooling with self-shaping material systems, in the design

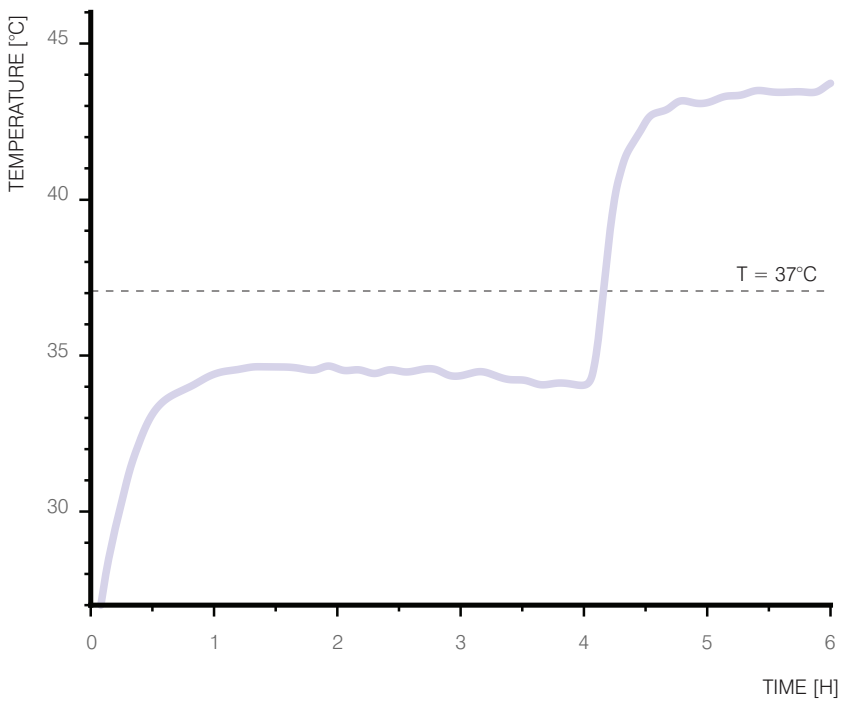

Figure 10: At temperatures above $32^{\circ} \mathrm{C}$, the PNIPAm-coated fleece releases moisture. Shown in the measured temperature profile curve, the developed passive cooling device will cool down its surroundings for a duration of about 3.5 hours.

of an on-body patch that changes shape to lower or lift the passive cooling element directly on and off the skin (Figure 9). After the passive cooling effect has been exhausted, the device can be recharged by soaking in water for 10 minutes until returning to its flat state and regaining enough moisture for reuse.

We developed a passive cooling element in-house. The passive cooling element was constructed from fleece wound dressings coated with 66 wt.\% poly( $N$-isopropyl acrylamide). As PNIPAm takes up water at temperatures below $32^{\circ} \mathrm{C}$ and releases it above $32^{\circ} \mathrm{C}$, the PNIPAm-coated fleece wound dressings exhibit passive cooling effects due to water evaporation (Figure 10). Specimens were measured using a thermal imaging camera over the course of irradiation by an infrared lamp. These experiments showed that temperatures were successfully lowered to a plateau of $33-34^{\circ} \mathrm{C}$, lasting for a duration of about 3.5 hours and obtaining significant cooling (in contrast, an uncoated wet wound dressing exceeded the cooling temperature of $33^{\circ} \mathrm{C}$ after less than 15 minutes).

We then incorporate this novel, but un-printable, passive cooling element into the FFF process. The fabrication sequence involves extruding the mesostructure while leaving voids for the later insertion of the passive cooling material. The voids have depths equal to the height of the external component; in addition to acting as placement indicators, they allow the passive cooling material to be inserted flush with the already extruded mesostructure and form a level surface for the continued extrusion of the remaining paths. We adjusted the extrusion parameters surrounding the wound dressing so that the passive cooling material is properly exposed, providing full contact with the skin, while being securely embedded in the mesostructure. 


\subsection{Design demonstrator}

Finally, we produce a prototype of a wearable orthotic splint that highlights the integration of all the presented functions in a highly customized end product, shown in Figure 11.

The device contains tailored areas of softness, as well as shape changing bumps to release pressure in sensitive areas when activated by moisture caused by sweating (Figure 11b). To limit movement, the splint can be inserted with an alignment tool to produce the required amount of immobilization (Figure 11d). Furthermore, multiple magnets are embedded at the edges to hold the splint closed.

This splint was designed to conform to a specific user and must wrap around an irregular wrist-forearm geometry. Without utilizing a 3D-scanner or any sophisticated inverse design algorithm, various material property gradients (Figure 11a) were tested for their ability to form and fit snugly around the non-uniform limb.

The use of flexible filament in the passive region was especially pivotal for expanding the range of achievable properties. As an intensive property of the material, the elastic modulus cannot be adapted; however, geometry can be used to additionally tune the stiffness (Figure 11c), resulting in mesostructures ranging from soft (allowing the splint to be bent from an initially flat state) to hard (providing support or immobilization).

We were able to quickly produce several functionally patterned iterations, and easily fabricate different designs until arriving at a differentiated mesostructure which allowed the device to be worn comfortably. The entire process, from initial design to final prototype, was completed over one weekend.

\section{CONCLUSION}

We have presented a design and material programming framework for creating 4D-printed material systems with differentiated and heterogeneous mesostructures. Directly designing mesostructures to achieve specific shape and behavior is tedious, difficult, and typically requires several full design iterations. Our approach provides a hierarchical workflow, which makes it simple and intuitive to rapidly iterate designs with complex self-shaping properties, by employing the intuitive geometric descriptions of existing CAD workflows for assigning and tuning multiple material behaviors throughout a volume. This framework can be used with commonly available, off-the-shelf 3D-printers - yet it also enables high customization by allowing non-printable components to be directly embedded in the end-product.

We have demonstrated this design framework through a series of case studies. Most notably, we showed the process of designing and fabricating an adaptive, orthotic splint (where interfacing with the body is rarely static) with highly intricate multi-material organization. Our presented approach abstracts the complexity of $4 \mathrm{D}$-printed mesostructures, and we used these internal geometries to tailor the splint to the unique wrist anatomy. To provide comfort, we modulated soft and compliant areas of the design through the tuning of mechanical properties in the passive regions. To prevent pressure peaks, we designed shape changing behaviors to allow adaptation over time in sensitive areas, using the active regions. Finally, we included a passive cooling effect by integrating a cutting edge but not yet printable material into the fabrication process.
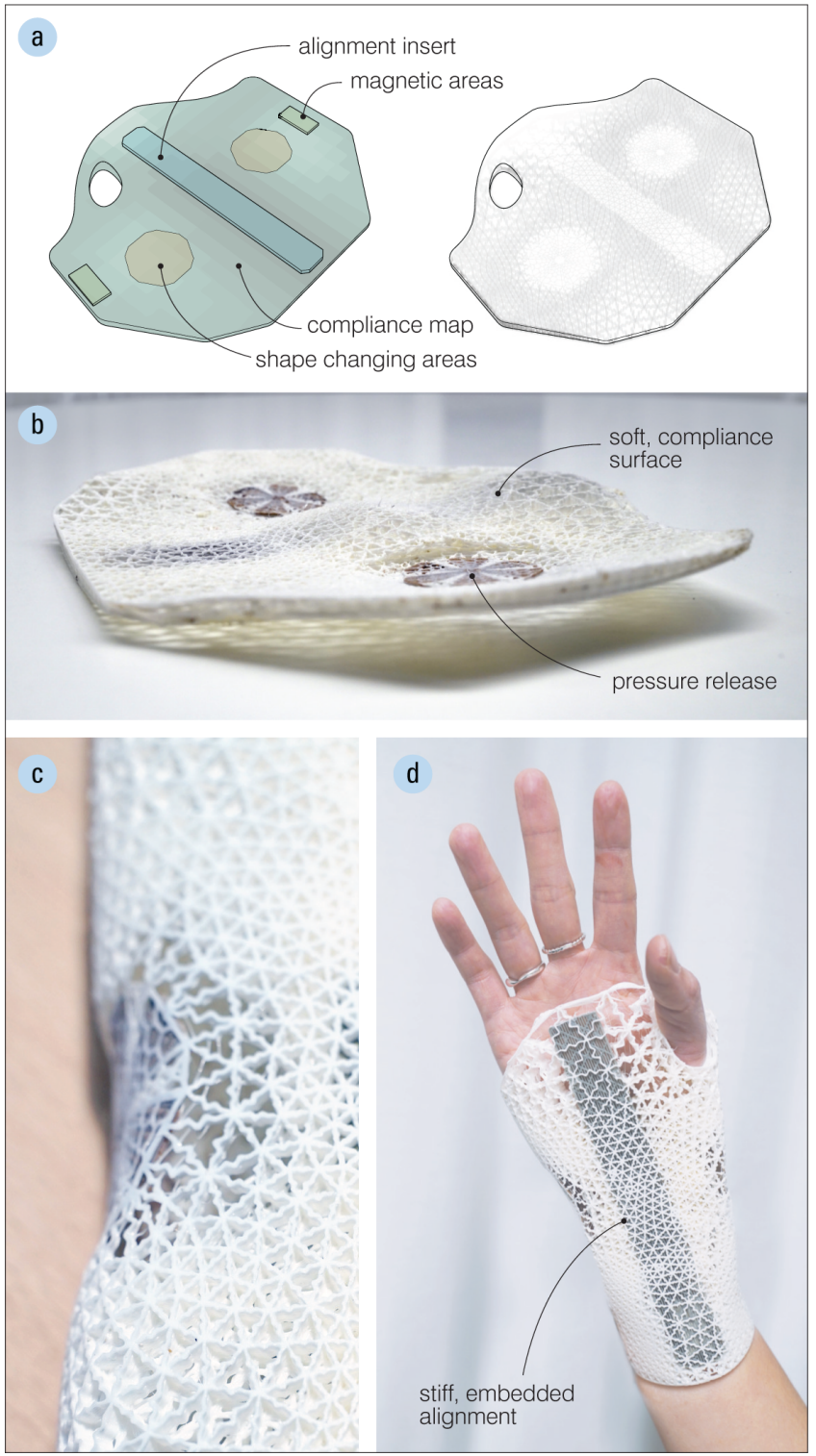

Figure 11: (a) The design and material programming of a multifunctional orthotic splint. (b) The 4D-printed splint with shape changing points for pressure release and the distribution of hard and soft areas. (c) Detail of the 4D-printed mesostructure. (d) The orthotic splint as worn by the user.

\subsection{Limitations and future work}

While this approach demonstrates the potential for designing sophisticated, functional, self-shaping systems, there are still several opportunities for further investigation. The layer-by-layer extrusion-based process of FFF has made it easy for us to tailor anisotropy in each layer. As we have only focused on designing functional gradation in the XY-plane, our results have been limited to surface geometries. 
At the same time, this method has made it possible to manage the complexity of functional structures and produce them with good mechanical and surface properties. We are particularly interested in expanding this framework for more design inputs in the Z-axis, while still taking advantage of FFF in its ability to easily produce anisotropy. Future explorations could include more spatial, volumetric properties and behaviors, such as the overlaying of different functions and textures on each side of the surface (for example, creating softness on the inside and roughness on the outside).

\subsection{Outlook}

We propose that the representation of objects by function and evolving in response to its surroundings (rather than by boundary and existing only as a predefined, singular geometry) adds value to AM. This ability to embed transformation and behaviors opens up the design space for many applications. By producing function through structure, we can capitalize on the potential of AM's relatively lowcost economies of scope to create variety and mass-customization. We posit that combining design across spatial-temporal scales with rapid design iteration (without the constraint of economies of scale from conventional manufacturing processes) will propel AM from being a rapid prototyping tool to a manufacturing platform.

\section{ACKNOWLEDGMENTS}

This work was funded by Baden-Württemberg Stiftung, through the "Innovation durch Additive Fertigung" research program [IAF-2 4DmultiMATS]; additional support has been provided by Deutsche Forschungsgemeinschaft, under the Cluster of Excellence IntCDC Integrative Computational Design and Construction in Architecture at the University of Stuttgart [EXC 2120/1 - 390831618]. We are grateful to Prof. Thomas Speck, Dr. Marc Thielen, Dr. Simon Poppinga, and Dr. Steve Heim for their insightful comments and discussion. We also thank Mobin Moussavi and Ahmad Razavi for their help with developmental tasks which contributed to this work.

\section{REFERENCES}

Alfredo Ferreira, Jr., Manuel J. Fonseca, and Joaquim A. Jorge. 2003. Polygon Detection from a Set of Lines. In $12^{\circ}$ Encontro Português de Computação Gráfica (EPCG). 159162.

Davide Jose Nogueira Amorim, Troy Nachtigall, and Miguel Bruns Alonso. 06162019 Exploring mechanical meta-material structures through personalised shoe sole design. In Proceedings of the ACM Symposium on Computational Fabrication, Stephen N. Spencer, James McCann, and Lining Yao (Eds.). ACM, New York, NY, USA, 1-8 https://doi.org/10.1145/3328939.3329001

Byoungkwon An, Hsiang-Yun Wu, Teng Zhang, Lining Yao, Ye Tao, Jianzhe Gu, Tingyu Cheng, Xiang 'Anthony' Chen, Xiaoxiao Zhang, Wei Zhao, Youngwook Do, and Shigeo Takahashi. 2018. Thermorph. In Proceedings of the 2018 CHI Conference on Human Factors in Computing Systems - CHI '18, Regan Mandryk, Mark Hancock, Mark Perry, and Anna Cox (Eds.). ACM Press, New York, New York, USA, 1-12. https://doi.org/10.1145/3173574.3173834

J. William Boley, Wim M. van Rees, Charles Lissandrello, Mark N. Horenstein, Ryan L. Truby, Arda Kotikian, Jennifer A. Lewis, and L. Mahadevan. 2019. Shape-shifting structured lattices via multimaterial $4 \mathrm{D}$ printing. Proceedings of the National Academy of Sciences 116, 42 (2019), 20856-20862. https://doi.org/10.1073/pnas. 1908806116

David Correa, Athina Papadopoulou, Christophe Guberan, Nynika Jhaveri, Steffen Reichert, Achim Menges, and Skylar Tibbits. 2015. 3D-Printed Wood: Programming Hygroscopic Material Transformations. 3D Printing and Additive Manufacturing 2, 3 (2015), 106-116. https://doi.org/10.1089/3dp.2015.0022

David Correa, Simon Poppinga, Max D. Mylo, Anna S. Westermeier, Bernd Bruchmann Achim Menges, and Thomas Speck. 2020. 4D pine scale: biomimetic 4D printed autonomous scale and flap structures capable of multi-phase movement. Philosophical transactions. Series A, Mathematical, physical, and engineering sciences 378, 2167 (2020), 20190445. https://doi.org/10.1098/rsta.2019.0445
Colin Dawson, Julian F. V. Vincent, and Anne-Marie Rocca. 1997. How pine cones open. Nature 390, 6661 (1997), 668. https://doi.org/10.1038/37745

Tobias Frenzel, Muamer Kadic, and Martin Wegener. 2017. Three-dimensional mechanical metamaterials with a twist. Science 358, 6366 (2017), 1072-1074. https://doi.org/10.1126/science.aao4640

Pedro Giachini, Sachin Gupta, Wendong Wang, Dylan Wood, Muhammad Yunusa, Ehsan Baharlou, Metin Sitti, and Achim Menges. 2020. Additive manufacturing of cellulose-based materials with continuous, multidirectional stiffness gradients. Science advances 6, 8 (2020), eaay0929. https://doi.org/10.1126/sciadv.aay0929

A. Sydney Gladman, Elisabetta A. Matsumoto, Ralph G. Nuzzo, L. Mahadevan, and Jennifer A. Lewis. 2016. Biomimetic 4D printing. Nature materials 15, 4 (2016), 413-418. https://doi.org/10.1038/nmat4544

William M. Harlow, Wilfred A. Côté, and A. Colin Day. 1964. The Opening Mechanism of Pine Cone Scales. Fournal of Forestry 62, 8 (1964), 538-540. https://doi.org/10. 1093/jof/62.8.538

Larry L. Howell. 2013. Compliant Mechanisms. In 21st Century Kinematics, J. Michael McCarthy (Ed.). Vol. 40. Springer London, London, 189-216. https://doi.org/10. 1007/978-1-4471-4510-3\{\}7

Alexandra Ion, Johannes Frohnhofen, Ludwig Wall, Robert Kovacs, Mirela Alistar, Jack Lindsay, Pedro Lopes, Hsiang-Ting Chen, and Patrick Baudisch. 10162016. Metamaterial Mechanisms. In Proceedings of the 29th Annual Symposium on User Interface Software and Technology, Jun Rekimoto, Takeo Igarashi, Jacob O. Wobbrock, and Daniel Avrahami (Eds.). ACM, New York, NY, USA, 529-539. https://doi.org/ 10.1145/2984511.2984540

Eline Kolken and Amir Zadpoor. 2017. Auxetic mechanical metamaterials. RSC Advances 7, 9 (2017), 5111-5129. https://doi.org/10.1039/C6RA27333E

Riccardo La Magna and Jan Knippers. 2018. Tailoring the Bending Behaviour of Material Patterns for the Induction of Double Curvature. In Humanizing Digital Reality, Klaas de Rycke, Christoph Gengnagel, Olivier Baverel, Jane Burry, Caitlin Mueller, Minh Man Nguyen, Philippe Rahm, and Mette Ramsgaard Thomsen (Eds.). Vol. 31. Springer Singapore, Singapore, 441-452. https://doi.org/10.1007/978-98110-6611-5\{_\}38

Thomas F. Lang, Adrian D. Leblanc, Harlan J. Evans, and Ying Lu. 2006. Adaptation of the proximal femur to skeletal reloading after long-duration spaceflight. Fournal of bone and mineral research : the official journal of the American Society for Bone and Mineral Research 21, 8 (2006), 1224-1230. https://doi.org/10.1359/jbmr.060509

Jennifer A. Lewis, Michael A. Bell, Travis A. Busbee, and John E. Minardi. 2016. Printed three-dimensional (3D) functional part and method of making.

Hod Lipson and Melba Kurman. 2013. Fabricated: The new world of $3 D$ printing. John Wiley and Sons, Indianapolis, Ind.

John C.S. McCaw and Enrique Cuan-Urquizo. 2018. Curved-Layered Additive Manufacturing of non-planar, parametric lattice structures. Materials \& Design 160 (2018), 949-963. https://doi.org/10.1016/j.matdes.2018.10.024

Vittorio Megaro, Jonas Zehnder, Moritz Bächer, Stelian Coros, Markus Gross, and Bernhard Thomaszewski. 2017. A computational design tool for compliant mechanisms. ACM Transactions on Graphics 36, 4 (2017), 1-12. https://doi.org/10.1145/3072959. 3073636

Achim Menges and Steffen Reichert. 2015. Performative Wood: Physically Programming the Responsive Architecture of the HygroScope and HygroSkin Projects. Architectural Design 85, 5 (2015), 66-73. https://doi.org/10.1002/ad.1956

Panagiotis Michalatos and Andrew Payne. 2016. Monolith: the biomedical paradigm and the inner complexity of hierarchical material design. In Complexity \& SimplicityProceedings of the 34th eCAADe Conference, Vol. 1. 445-454.

Laia Mogas-Soldevila, Jorge Duro-Royo, and Neri Oxman. 2014. Water-Based Robotic Fabrication: Large-Scale Additive Manufacturing of Functionally Graded Hydrogel Composites via Multichamber Extrusion. 3D Printing and Additive Manufacturing 1, 3 (2014), 141-151. https://doi.org/10.1089/3dp.2014.0014

Matthias Müller, Bruno Heidelberger, Matthias Teschner, and Markus Gross. 2005. Meshless deformations based on shape matching. ACM Transactions on Graphics 24, 3 (2005), 471-478. https://doi.org/10.1145/1073204.1073216

Neri Oxman. 2010. Structuring Materiality: Design Fabrication of Heterogeneous Materials. Architectural Design 80, 4 (2010), 78-85. https://doi.org/10.1002/ad.1110

Julian Panetta, Qingnan Zhou, Luigi Malomo, Nico Pietroni, Paolo Cignoni, and Denis Zorin. 2015. Elastic textures for additive fabrication. ACM Transactions on Graphics 34, 4 (2015), 1-12. https://doi.org/10.1145/2766937

Simon Poppinga, Nikolaus Nestle, Andrea Šandor, Bruno Reible, Tom Masselter, Bernd Bruchmann, and Thomas Speck. 2017. Hygroscopic motions of fossil conifer cones. Scientific reports 7 (2017), 40302. https://doi.org/10.1038/srep40302

Jordan R. Raney and Jennifer A. Lewis. 2015. Printing mesoscale architectures. Mrs Bulletin 40, 11 (2015), 943-950. https://doi.org/10.1557/mrs.2015.235

Etienne. Reyssat and Lakshminarayanan Mahadevan. 2009. Hygromorphs: from pine cones to biomimetic bilayers. Fournal of the Royal Society, Interface 6, 39 (2009), 951-957. https://doi.org/10.1098/rsif.2009.0184

K. E. Rudman, Richard Aspden, and Judith Meakin. 2006. Compression or tension? The stress distribution in the proximal femur. Biomedical engineering online 5 (2006), 12. https://doi.org/10.1186/1475-925X-5-12 
Laxminarayana Saggere and Sridhar Kota. 1999. Static Shape Control of Smart Structures Using Compliant Mechanisms. AIAA fournal 37, 5 (1999), 572-578. https://doi.org/10.2514/2.775

Skylar Tibbits. 2014. 4D Printing: Multi-Material Shape Change. Architectural Design 84, 1 (2014), 116-121. https://doi.org/10.1002/ad.1710

Alexander D. Valentine, Travis A. Busbee, John William Boley, Jordan R. Raney, Alex Chortos, Arda Kotikian, John Daniel Berrigan, Michael F. Durstock, and Jennifer A. Lewis. 2017. Hybrid 3D Printing of Soft Electronics. Advanced materials (Deerfield Beach, Fla.) 29, 40 (2017). https://doi.org/10.1002/adma.201703817

Elena Vazquez, Benay Gürsoy, and Jose Pinto Duarte. 2020. Formalizing shapechange: Three-dimensional printed shapes and hygroscopic material transformations. International Journal of Architectural Computing 18, 1 (2020), 67-83. https://doi.org/10.1177/1478077119895216

Wen Wang, Lining Yao, Teng Zhang, Chin-Yi Cheng, Daniel Levine, and Hiroshi Ishii. 2017. Transformative Appetite. In Proceedings of the 2017 CHI Conference on Human
Factors in Computing Systems. Association for Computing Machinery, New York, NY, USA, 6123-6132. https://doi.org/10.1145/3025453.3026019

Julius Wolff. 1870. Ueber die innere Architectur der Knochen und ihre Bedeutung für die Frage vom Knochenwachsthum. Archiv für Pathologische Anatomie und Physiologie und für Klinische Medicin 50, 3 (1870), 389-450. https://doi.org/10.1007/BF01944490

Dylan Marx Wood, David Correa, Oliver David Krieg, and Achim Menges. 2016. Material computation-4D timber construction: Towards building-scale hygroscopic actuated, self-constructing timber surfaces. International fournal of Architectural Computing 14, 1 (2016), 49-62. https://doi.org/10.1177/1478077115625522

Lining Yao, Jifei Ou, Chin-Yi Cheng, Helene Steiner, Wen Wang, Guanyun Wang, and Hiroshi Ishii. 2015. bioLogic. In Proceedings of the 33rd Annual ACM Conference on Human Factors in Computing Systems - CHI '15, Bo Begole, Jinwoo Kim, Kori Inkpen, and Woontack Woo (Eds.). ACM Press, New York, New York, USA, 1-10. https://doi.org/10.1145/2702123.2702611 